\title{
Advances in Fine Needle Aspiration Cytology for the Diagnosis of Pulmonary Carcinoma
}

\author{
Adnan Hasanovic, Natasha Rekhtman, Carlie S. Sigel, and Andre L. Moreira \\ Department of Pathology, Memorial Sloan-Kettering Cancer Center, 1275 York Avenue, \\ New York, NY 10065, USA \\ Correspondence should be addressed to Andre L. Moreira, moreiraa@mskcc.org
}

Received 5 February 2011; Accepted 3 May 2011

Academic Editor: Darshana Jhala

Copyright ( $\odot 2011$ Adnan Hasanovic et al. This is an open access article distributed under the Creative Commons Attribution License, which permits unrestricted use, distribution, and reproduction in any medium, provided the original work is properly cited.

\begin{abstract}
New developments in the field of thoracic oncology have challenged the way pathologists approach the diagnosis of pulmonary carcinoma. Nonsmall cell carcinoma is no longer an adequate diagnostic category. Pathologists are required to further classify tumors into adenocarcinoma and squamous cell carcinoma since specific therapies are now recommended depending on the histological tumor type. This change occurred following the discovery of specific molecular alterations that predict response to certain drugs and now molecular testing of tumor cells is often requested to direct therapy. The vast majority of lung cancer is diagnosed in advanced clinical stages, where cytologic or small biopsy material is the only form of tissue diagnosis, thus placing cytology, especially fine needle aspiration biopsy in the front line for management of patients with lung cancer. In this paper we will review the current concepts in the suitability and accuracy of fine needle aspiration biopsy, including diagnosis, classification, prognostic markers, and use of ancillary techniques.
\end{abstract}

\section{Introduction}

Pulmonary nodules discovered by an imaging technique present a relatively frequent clinical problem. A solitary pulmonary nodule is a common manifestation of a benign condition. However, in nodules larger than $2 \mathrm{~cm}$, the incidence of a primary lung cancer ranges from 64 to $82 \%$ [1]. An early, accurate diagnosis is of paramount importance for initiating specific therapy for malignant lesions, and for avoiding unnecessary procedures for benign conditions. Thus, after clinical risk assessment tissue diagnosis is the next step in managing radiologically suspicious lung nodules. Direct tissue sampling for diagnosis is essential in most patients for decisions regarding treatment and can be accomplished by fine needle aspiration biopsy (FNAB), endoscopic or core needle biopsy, or surgical resection. Sampling of the lesion by FNAB can be performed via the airway (endobronchial transbronchial FNAB) or chest wall (CT-guided percutaneous FNAB). Transbronchial FNAB is useful for the diagnosis of primary pulmonary lesions that lie beneath the bronchial surface and for staging lung cancer patients by sampling mediastinal lymph nodes. FNAB has become recognized as a safe and effective diagnostic tool, as a result of improved aspiration biopsy tools and techniques, better control of complications, and increased experience of cytopathologists in interpreting aspirate specimens.

Most patients with lung cancer present with clinical advanced disease and therefore are not candidates for surgery with curative intent, but are rather treated with systemic therapies. In the age of personalized therapies, cytological material in the form of FNAB may be the only available diagnostic specimen, and the only material available for molecular studies, necessary for current therapeutic decision making. New recommendations for screening of high-risk populations [2] coupled with the ongoing development of minimally invasive techniques and procedures for sampling lung lesions will most likely further increase the need for accurate diagnosis and molecular characterization of malignant tumors on small biopsy specimens.

In this paper, we will cover current concepts and advances in FNAB of pulmonary carcinomas including diagnosis, 

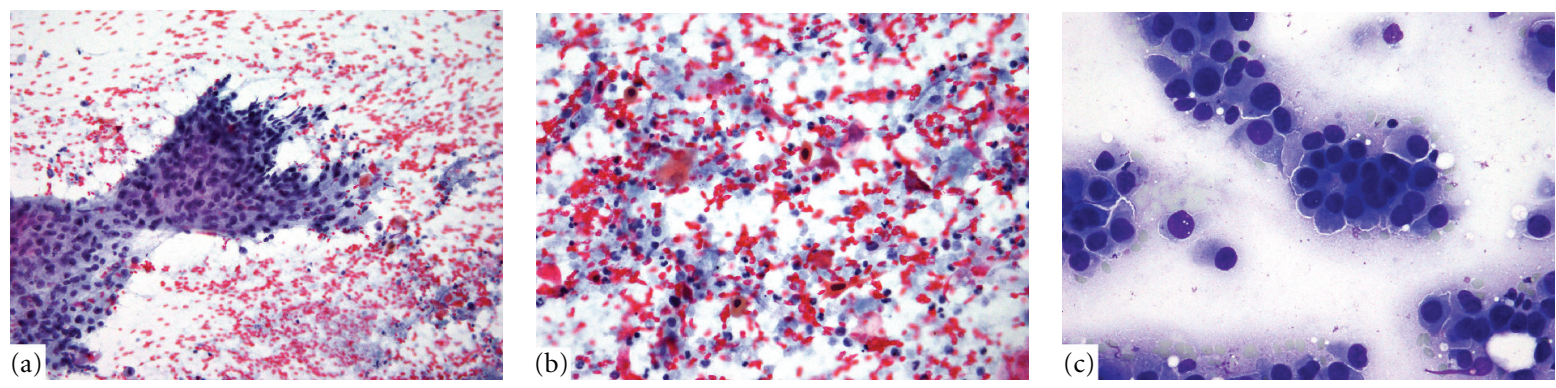

FIGURE 1: The characteristic morphologic patterns of tumor in smears. (a) SQC carcinoma showing flat sheet of polygonal, atypical cells and (b) orangeophilia on Pap stain demonstrating keratinization. (c) ADC with typical cytomorphology and formation of glandular structures.

classification, prognostic makers, and use of ancillary techniques.

\section{Clinical Advances in the Management of Patients with Pulmonary Carcinoma}

The current classification of lung cancer recognizes four major histological subtypes, namely, squamous cell carcinoma (SQC), adenocarcinoma (ADC), large-cell carcinoma (LCC), and small cell lung carcinoma (SCLC). Until recently, most of the cytological diagnosis of lung carcinoma was based on distinguishing SCLC from other tumors generally designated as nonsmall cell carcinoma (NSCLC), because these two categories were the most relevant for directing therapy. However, advances in thoracic medical oncology have led to a paradigm shift in NSCLC diagnosis, resulting in a new emphasis on accurate NSCLC subtyping. Specifically, two novel agents have challenged NSCLC as clinically relevant diagnostic category. It has been demonstrated that patients with the diagnosis of SQC are at increased risk for life-threatening complications if treated with bevacizumab, a humanized antibody against vascular endothelial growth factor (VEGF) [3]. In addition, in the case of pemetrexed, an antifolate that inhibits multiple enzymes in purine and pyrimidine synthesis, patients with SQC showed no response to the drug in comparison to a good response observed in patients with the diagnosis of non-squamous cell carcinoma [4]. For these reasons, these two new drugs are only recommended for use in patients with a diagnosis of non-squamous cell carcinoma. Other developments include the identification of genetic alterations which have been described almost exclusively in adenocarcinoma that confer susceptibility to therapeutic agents or resistance to chemotherapeutic drugs. For example, tumors with epidermal growth factor (EGFR) mutations have a better outcome and respond to the tyrosine kinase inhibitors erlotinib and gefitinib, as a first-line therapy, whereas patients without EGFR mutations seem to have a better outcome with standard chemotherapy [5]. Furthermore, translocation in the EML4-ALK gene has been described predominantly in adenocarcinomas. This translocation confers susceptibility to specific inhibitor, crizotinib that is currently undergoing clinical testing [6].
These advances in the understanding of molecular mechanisms underlying lung cancer and the development of new targeted therapies challenge the traditional diagnostic dichotomization between SCLC and NSCLC and prompt a more specific characterization of NSCLC into squamous or adenocarcinoma category. Traditionally, NSCLC subclassification has been based on morphologic assessment of routine $\mathrm{H} \& \mathrm{E}$-stained histological specimens. Because cytology specimens, such as FNAB, differ in preparation and technique from traditional histology, the accuracy of subtyping these specimens has been challenged, yet there is considerable evidence supporting the utility of cytology in both subtyping NSCLC and providing material for predictive and prognostic studies.

\section{Role of Immediate Assessment in the Accuracy of Lung FNA Cytological Diagnosis}

Published reports reveal that the sensitivity of FNAB for the diagnosis of lung cancer ranges from 56 to over $90 \%$ whereas specificity is close to $100 \%$. In nearly all these studies, the overall positive predictive value is nearly $99 \%$. While the false positive rate is generally less than $1 \%$, a negative result is less reliable with most studies reporting a false negative rate of around $10 \%[7,8]$. The major contribution to the relatively high false negative rate is failure to obtain diagnostic material, most commonly due to sampling error. Studies have shown that immediate onsite assessment is valuable in minimizing false negative diagnoses due to nondiagnostic material $[9,10]$. Published series by Austin and Cohen show that immediate on-site assessment during FNAB was associated with a statistically significant increased diagnostic accuracy compared to cases without immediate assessment, $100 \%$ versus $80 \%$, respectively [11]. During on site adequacy determination, smears from the aspirate are rapidly stained and are evaluated by a cytopathologist or cytotechnologist for cellularity and diagnostic yield. On-site adequacy evaluation also provides real-time communication of information including appropriate tissue triage recommendations for ancillary tests such as molecular testing, flow cytometry, cytogenetics, electron microscopy, and so forth. This interaction directly impacts clinical management during the critical 
TABLE 1: Factors contributing to difficulty of cytologic subtyping of adenocarcinoma and squamous cell carcinoma.

\begin{tabular}{lccccc}
\hline$N=165$ & \multicolumn{2}{c}{ Cellularity } & \multicolumn{2}{c}{ Differentiation $^{(1)}$} & \multicolumn{2}{c}{ Histologic type } \\
& Low & High & Well-moderately & Poorly & ADC \\
\hline Correct definitive Subtyping $(n=148)$ & $82 \%$ & $94 \%$ & $87 \%$ & $69 \%$ & $93 \%$ \\
Difficulty in Subtyping $(n=17)^{(2)}$ & $18 \%$ & $6 \%$ & $13 \%$ & $31 \%$ & $70 \%$ \\
\hline
\end{tabular}

${ }^{(1)}$ Grade of differentiation was based on resected specimens.

${ }^{(2)}$ Difficulty in subtyping encompasses cases which were incorrectly classified, unclassified, or underclassified/subtype favored by cytology.

diagnostic phase while the lesion can still be readily sampled.

\section{Accurate Morphological Diagnosis of Subtypes of NSCLC}

Morphology still remains the cornerstone in lung cancer classification. The World Health Organization classification of lung tumors through the 1999 edition did not address lung cancer diagnosis based on small biopsies and cytology [12]. In the 2004 World Health Organization classification, cytology was addressed for the first time, with descriptions of the morphological criteria for each type of pulmonary carcinoma [13]. In the new revised proposal [14], an entire section is dedicated to the classification of lung tumors based on small biopsy material including FNAB. This highlights the importance and recognition of the role that FNAB plays in the diagnosis and management of pulmonary carcinomas.

Lung cancer histological subtypes that are morphologically recognizable on cytology specimens are ADC, SQC, and SCLC, as well as carcinoid tumors. Other types of lung carcinoma such as large cell carcinoma and other rare variant as fetal type and colloid adenocarcinoma may be suspected on the basis of pure morphology but usually require evaluation of the surgically resected specimen for the final diagnosis.

Historically, it has been important to accurately identify SCLC as the treatment is different from NSCLC. Classical morphological features of SCLC such as nuclear molding, frequent mitoses, and absence of nucleoli are often distorted on a small biopsy specimen showing extensive crush artifact. In this setting, cytology has an edge over histology because of better preservation and fewer artifacts [15]. Cytology is highly accurate and a well-recognized method to distinguish SCLC from NSCLC. In a study of 259 consecutive lung FNAs by Delgado et al., SCLC was distinguished from NSCLC with accuracy of $96 \%$ [16].

Unlike the distinction of SCLC and NSCLC, feasibility of NSCLC subtyping in cytology has been controversial. However, based on a recent study from our institution, cytology provides several advantages over surgical specimens for the subtyping of NSCLC [17]. The key morphologic criteria for ADC versus SQC are glandular architecture versus keratinization, respectively. The Papanicolaou (Pap) stain has exquisite sensitivity for even minimal keratinization aiding in the distinction of SQC from ADC. The morphologic patterns which emerge in tumor smears provide a clue to a tumor subtype which may not be apparent in surgical
TABLE 2: Sensitivity and specificity of cytologic tumor subtyping.

\begin{tabular}{lccc}
\hline$n=183$ & Sensitivity & Specificity & Accuracy \\
\hline SQC versus non-SQC & $87 \%$ & $98 \%$ & $97 \%$ \\
ADC versus non-ADC & $98 \%$ & $79 \%$ & $93 \%$ \\
\hline
\end{tabular}

specimens (Figure 1). In addition, due to immediate fixation, cytology provides greater nuclear and cytoplasmic resolution than histology. While in the majority of cases a line of differentiation can be clearly identified by morphology, difficulties arise in a subset of cases.

In a recent study, which included 165 cases with paired FNAB and resection diagnosis of ADC and SQC, we described some of the limiting factors for the interpretation and accurate classification in cytology specimens. The strongest predictors for difficulty in subtyping were poor differentiation of the tumor where distinguishing morphologic features are not apparent (Table 1), followed by scant cellularity. Nonkeratinizing poorly differentiated squamous cell carcinoma in particular is subject to misclassification by FNAB [18]. Another difficulty is presented by tumors with mixed histology, but true adenosquamous carcinomas are infrequent with reported incidence of 2 to $3 \%$ in published surgical series [19].

Despite these limitations, using morphology and occasional immunocytochemistry we observed that when faced with the need to subclassify NSCLC we performed with high concordance between cytology and resected specimens of $97 \%$ and $93 \%$ for identifying squamous and adenocarcinoma, respectively (Table 2). Despite the lower sensitivity for non-keratinizing SQC, the specificity of this diagnosis is very high, which means that the false-positive classification as SQC is extremely rare. Thus having proved overall high accuracy of cytology in distinguishing SQC versus non-SQC it was concluded that cytological specimens are suitable for guiding therapeutic decisions within these diagnostic categories [18].

\section{Use of Immunohistochemistry Stains in the Characterization of NSCLC Subtypes}

In tumors that do not show clear-cut signs of differentiation on light microscopy examination (Figure 2) further investigation by immunohistochemistry may highlight tumor cell lineage. In biopsies like FNAB, with limited material, the need to conserve material for possible mutational analysis and other prognostic markers obligates the use of the most 


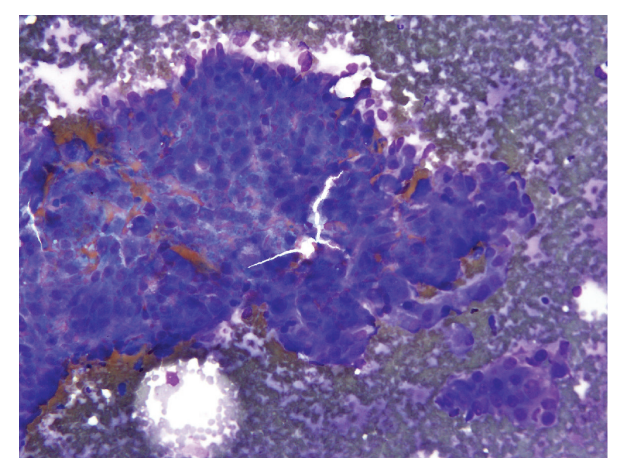

Figure 2: Poorly differentiated carcinoma lacking clear signs of differentiation on light microscopic examination. Further work up using limited panel of antibodies will differentiate tumor cell lineage in the majority of cases.

efficient and limited panel of immunohistochemical stains. Several recent publications have addressed the question of what is the best panel of markers to be used in the distinction between adenocarcinomas and squamous cell carcinoma. Results published by $\mathrm{Wu}$ et al. showed the advantages of immunocytochemistry in distinguishing poorly differentiated SQC from SCLC of the lung, and primary adenocarcinoma from metastatic tumors in cytology specimens. In their study, all poorly differentiated squamous cell carcinomas showed expression of p63 while negative for thyroid transcription factor-1 (TTF-1), whereas adenocarcinomas had the opposite staining pattern. Of note, SCLC had identical p63/TTF-1 expression profile as adenocarcinoma, but application of standard morphologic criteria and addition of neuroendocrine markers was sufficient for accurate classification [20]. Nicholson et al., in their recently published work that included 13 FNA cytology specimens, showed that a limited panel of TTF-1, CK5/6, and p63 together with a mucin stain, refined diagnosis of NSCLC to either ADC or SQC in 65\% of cases [21].

In summary, most of the work has been concentrated on the expression of 3 markers TTF-1, p63, and a highmolecular-weight cytokeratin (HMWCK). The following algorithm, based on published data and our experience can guide the interpretation of the stains. A TTF-1 negative/p63positive/HMWCK-positive profile is supportive of SQC, whereas any expression of TTF- 1 is supportive of ADC. It is worth mentioning that coexpression of TTF- 1 and p 63 can be seen in adenocarcinomas. In most cases p63 expression in adenocarcinoma is patchy and weak. Negative staining for both p63 and TTF-1 usually rules out squamous cell carcinoma [22].

Napsin-A is an aspartic proteinase, involved in the maturation of the surfactant protein B and is expressed in the cytoplasm of cells of lung and kidney [23]. The staining is cytoplasmic and is strongly positive in up to $80 \%$ of primary lung adenocarcinomas. In the study of Stoll et al., 75 cytology cases were analyzed. It showed that the sensitivity and specificity of TTF-1 were each $81 \%$. Napsin-A exceeded the specificity of TTF- 1 at $96 \%$ with a lower sensitivity of $65 \%$. In the study, the only carcinoma of nonlung origin in which Napsin-A was detected was renal cell carcinoma, suggesting that Napsin-A can be used as a surrogate marker in work up of poorly differentiated lung adenocarcinoma or an unknown primary tumor [24]. Desmocolin-3, constitutive protein of desmosomes, is found to be overexpressed in SQC of the lung $[25,26]$. In the study by Monica et al., which included 31 cytological specimens originally classified as NSCLC, staining for desmocolin-3 and TTF-1 was mutually exclusive in tumors [26].

Several authors reported on the high sensitivity and specificity of miRNA expression in SQC of the lung, and its usefulness in differentiating ADC from SQC in small specimens $[27,28]$. However, a recently published study does not support this observation [29]. The utility of these new approaches compared to standard markers needs to be evaluated further and validated, since a limited panel of immunohistochemical markers can reproduce the results obtained by the new molecular techniques [22].

\section{Adequacy of Material Obtained by FNAB for Molecular Testing}

In the recent years we have witnessed a revolution in our understanding of the molecular basis of NSCLC. These advances have led to development of multitude of commercially available prognostic and predictive biomarkers and targeted therapeutic agents. Despite these advances in treatment, the overall prognosis remains poor in patients with advanced disease. Personalizing therapy based on an individual tumor molecular profile can optimize efficacy with the available agents. Molecular determinants that guide treatment decision-making may have a prognostic or predictive function, and are commonly referred to as prognostic or predictive markers, respectively. Prognostic marker refers to a tumor characteristic that is useful for estimating a patient's outcome independent of therapeutic decisions. In contrast, predictive markers are useful in making therapeutic decisions. Mutations of EGFR, KRAS, and EML4/ALK translocations are mutually exclusive in lung $\mathrm{ADC}$ and identify tumor subsets with unique dependencies and drug sensitivities. KRAS mutation testing is utilized by some institutions to exclude EGFR mutations or an EML4/ALK translocation.

In particular EGFR mutation and EML4/ALK fusion gene testing have reached clinical validation and are incorporated into the current treatment paradigm [30]. EML4/ALK testing also appears to have clinical utility in identifying patients who could benefit from referral for a study targeting $A L K$ inhibition, such as ongoing phase 3 studies of the small molecule tyrosine kinase inhibitor crizotinib.

There is increasing awareness that the quality of specimens, such as cytology, has a profound influence on molecular diagnostic test results. FNAB samples are exposed to a greater variety of cytopreparation methods than resected tissue and sample size and heterogeneity may have an effect on the downstream molecular test results [31]. Most molecular techniques including in situ hybridization, polymerase chain reaction (PCR), and transcriptional profiling can be done on FNAB specimens $[32,33]$. Optimizing and standardizing 
of FNAB sample preparation methods is needed to preserve biomolecular integrity to enable seamless integration into molecular testing.

At present, there are few studies that rigorously compare the cellular composition of FNA samples with the quantity and quality of the desired analyte (DNA, mRNA, or protein) or the robustness of the biomarker test utilizing the sample. Schuurbiers et al. in their study conclude that molecular testing of EGFR and KRAS on cytologic material obtained by endobronchial ultrasound-guided transbronchial FNAB is feasible and could be performed on $77 \%$ of their specimens [34]. Another study by Smouse et al. showed that $67 \%$ of cytology specimens were adequate for molecular testing with some of the samples having as little as $25 \%$ tumor cellularity [35].

We have found that in a large screen of various cytologic specimens (FNABs, effusions, and exfoliative specimens) submitted for EGFR or KRAS mutational testing, 98\% of samples were suitable for analysis. We concluded that testing is feasible and that with rare exception all cell blocks subjectively interpreted as "adequate" for diagnosis by a pathologist yielded sufficient quantity and quality of DNA for mutational analysis. This finding is in agreement with several other studies [18]. In a study of consecutive specimens from our institution, it was found that $79 \%$ of cytology specimens and $89 \%$ of small biopsy specimens submitted for molecular testing were sufficiently cellular [36]. The rate of EGFR and KRAS mutations detected in cytologic specimens in the study was comparable to the rate detected in surgical specimens.

\section{Use of FNAB for Prognostic Markers}

The reported incidence of local or distant recurrence following surgical resection of early-stage NSCLC is around $36 \%$ [37]. The risk of recurrence is clearly linked to clinical stage but further biomarkers predictive of tumor recurrence are needed. A histological grading system based on the predominant histological patterns seen in pulmonary adenocarcinoma has been described recently [38]. In that study, patients with stage 1 pulmonary adenocarcinoma could be accurately stratified according to risk of recurrence of death of the disease into 3 tiers representing low, intermediate and high risk, thus indicating that an objective system of tumor grade had prognostic significance. In cytological material however, there was no reliable corresponding pattern of cellular aggregates to predict a histological pattern for the same tumor [39], therefore a grading system based on nuclear features has been developed and evaluated in FNAB of pulmonary adenocarcinoma. The cytology grading system is based on the nuclear size of neoplastic cells; pattern of chromatin distribution; and nuclear contour and it separates stage 1 pulmonary adenocarcinomas into two groups. Tumors with low nuclear scores show a lower risk of recurrence or death of the disease in contrast to tumors with high nuclear scores [40]. In conclusion, FNAB can provide significant prognostic information to clinicians managing patients with pulmonary adenocarcinoma.

EGFR mutations are the best predictor of response to EGFR kinase inhibitors in pulmonary adenocarcinoma.
Recently, two antibodies that detect specifically mutated EGFR proteins have become commercially available. A recent study from our institution demonstrated that these antibodies show sensitivity of $95 \%$ for the detection of EGFR L858R mutation, and sensitivity of $85 \%$ for the detection of exon 19 deletions. They concluded that immunohistochemistry for mutated EGFR could be used as a screen method to identify candidates for therapy with EGFR tyrosine-kinase inhibitors [41].

An antibody that correlates with $A L K$ gene rearrangement in NSCLC has also been reported recently with promising results $[42,43]$ however this antibody is not yet commercially available.

The use of these antibodies in cytological material has not yet been validated, but they may offer an invaluable tool for screening patients with lung cancer where cytological material is the only specimen available.

\section{New Sampling Methods in Lung FNAB (EBUS and ENB)}

A couple of new minimally invasive procedures have been recently developed as an alternative to standard approaches. One of them, endobronchial ultrasound-guided transbronchial needle aspiration (EBUS-TBNA) has recently emerged as a valid minimally invasive method for mediastinal staging of lung cancers and diagnostic workup of centrally located masses [44]. When used to sample mediastinal lymph nodes, at least a moderate number of lymphocytes must be present to ensure the adequacy of the specimen and avoid a false negative result. A large retrospective study performed at our institution found that EBUS-TBNA had $89 \%$ sensitivity and $100 \%$ specificity for malignant disease, revealing no major discrepancies between tumor subtypes rendered by EBUS-TBNA cytology and histology [45]. A study by Turnoy et al. showed that in patients with NSCLC without extrathoracic metastasis, EBUS-TBNA reduces the need for surgical staging by $68 \%$ with lower incidence of complications and no difference in diagnostic performance, thus establishing the procedure as a valid alternative to mediastinoscopy [46].

The two major limitations of standard flexible bronchoscopy are its inability to reach peripheral segments of the lung and the limited diagnostic yield from lesions less than $3 \mathrm{~cm}$ in diameter. The alternative to bronchoscopy is CT-guided percutaneous biopsy where the possible complications include hemorrhage and pneumothorax. Recently developed technology that is emerging in clinical practice essentially combines these two methods. Electromagnetic navigation is a localization device that assists in placing endobronchial accessories (e.g., forceps, brush, and needle) in the desired areas of the lung. The system uses low frequency electromagnetic waves and real-time 3D digital reconstruction of the previously obtained CT scan of the bronchial tree. Electromagnetic navigational bronchoscopy (ENB) systems were recently cleared by the US Food and Drug Administration to aid the physician in guiding endoscopic tools in the respiratory tract. This novel technical advance localizes and samples lesions in the lung 
parenchyma and mediastinum that are beyond the reach of standard endoscopy [47]. Recent published studies showed the diagnostic yield of ENB for small peripheral lung lesions are in the range from $54 \%$ to $77 \%$. Lamprecht et al. studied ENB sampling using rapid on site cytological evaluation during the procedure and it showed sensitivity and specificity of $84.6 \%$ and $100 \%$, respectively. Citing potential drawbacks, they found that $33.3 \%$ of the cases with ENB sampling were falsely negative and definitive diagnosis had to be established by CT-guided biopsy or by surgery [48]. Of note, other authors have reported that presence of cytologist virtually eliminates the problem of inadequate samples $[49,50]$.

\section{Conclusion}

The field of thoracic oncology is going through a revolution with the advent of targeted therapy for the management of patients with lung cancer. FNAB is in many cases the only diagnostic specimen available for guiding therapeutic decisions. FNAB has proven to be an invaluable tool not only for diagnostic accuracy of pulmonary carcinomas classification, but also as a reliable and adequate source of material suitable for molecular analysis.

\section{Conflict of Interests}

The authors declare no conflict of interests.

\section{References}

[1] M. M. Wahidi, J. A. Govert, R. K. Goudar, M. K. Gould, and D. C. McCrory, "Evidence for the treatment of patients with pulmonary nodules: when is it lung cancer? ACCP evidencebased clinical practice guidelines (2nd edition)," Chest, vol. 132, no. 3, pp. 94S-107S, 2007.

[2] D. R. Aberle, C. D. Berg, W. C. Black et al., "The national lung screening trial: overview and study design," Radiology, vol. 258, no. 1, pp. 243-253, 2011.

[3] A. Sandler, "Bevacizumab in non-small cell lung cancer," Clinical Cancer Research, vol. 13, no. 15, part 2, pp. s4613s4616, 2007.

[4] G. Scagliotti, N. Hanna, F. Fossella et al., "The differential efficacy of pemetrexed according to NSCLC histology: a review of two phase III studies," Oncologist, vol. 14, no. 3, pp. 253263, 2009.

[5] J. L. Marks, S. Broderick, Q. Zhou et al., "Prognostic and therapeutic implications of EGFR and KRAS mutations in resected lung adenocarcinoma," Journal of Thoracic Oncology, vol. 3, no. 2, pp. 111-116, 2008.

[6] E. L. Kwak, Y. J. Bang, D. R. Camidge et al., "Anaplastic lymphoma kinase inhibition in non-small-cell lung cancer," The New England Journal of Medicine, vol. 363, no. 18, pp. 1693-1703, 2010.

[7] R. Nizzoli, M. Tiseo, F. Gelsomino et al., "Accuracy of fine needle aspiration cytology in the pathological typing of nonsmall cell lung cancer," Journal of Thoracic Oncology, vol. 6, no. 3, pp. 489-493, 2011.

[8] M. F. Zakowski, "Fine-needle aspiration cytology of tumors: diagnostic accuracy and potential pitfalls," Cancer Investigation, vol. 12, no. 5, pp. 505-515, 1994.

[9] C. J. R. Stewart and I. S. Stewart, "Immediate assessment of fine needle aspiration cytology of lung," Journal of Clinical Pathology, vol. 49, no. 10, pp. 839-843, 1996.
[10] C. P. Mullan, B. E. Kelly, P. K. Ellis, S. Hughes, N. Anderson, and W. G. McCluggage, "CT-guided fine-needle aspiration of lung nodules: effect on outcome of using coaxial technique and immediate cytological evaluation," Ulster Medical Journal, vol. 73, no. 1, pp. 32-36, 2004.

[11] J. H. M. Austin and M. B. Cohen, "Value of having a cytopathologist present during percutaneous fine-needle aspiration biopsy of lung: report of 55 cancer patients and metaanalysis of the literature," American Journal of Roentgenology, vol. 160, no. 1, pp. 175-177, 1993.

[12] W. D. Travis, B. Corrin, Y. Shimosato, and E. Brambilla, World Health Organization International Histological Classification of Tumours. Histological Typing of Lung and Pleural Tumours, Springer, 3rd edition, 1999.

[13] W. D. Travis, H. K. Muller-Hermelink, and C. C. Harris, World Health Organization Classification of Tumours. Pathology and Genetics of Tumours of the Lung, Pleura, Thymus and Heart, International Agency For Research On Cancer, Lyon, France, 2004.

[14] W. D. Travis, E. Brambilla, M. Noguchi et al., "International association for the study of lung cancer/American thoracic society/European respiratory society international multidisciplinary classification of lung adenocarcinoma," Journal of Thoracic Oncology, vol. 6, no. 2, pp. 244-285, 2011.

[15] M. F. Zakowski, "Pathology of small cell carcinoma of the lung," Seminars in Oncology, vol. 30, no. 1, pp. 3-8, 2003.

[16] P. I. Delgado, M. Jorda, and P. Ganjei-Azar, "Small cell carcinoma versus other lung malignancies: diagnosis by fine-needle aspiration cytology," Cancer, vol. 90, no. 5, pp. 279-285, 2000.

[17] C. S. Sigel, A. L. Moreira, W. D. Travis et al., "Subtyping of Non-Small Cell Lung Carcinoma (NSCLC): A Comparison of Small Biopsy and Cytology Specimens," Journal of Thoracic Oncology, (In press).

[18] N. Rekhtman, S. M. Brandt, C. S. Sigel et al., "Suitability of thoracic cytology for new therapeutic paradigms in non-small cell lung carcinoma: high accuracy of tumor subtyping and feasibility of EGFR and KRAS molecular testing," Journal of Thoracic Oncology, vol. 6, no. 3, pp. 451-458, 2011.

[19] J. Shimizu, M. Oda, Y. Hayashi, A. Nonomura, and Y. Watanabe, "A clinicopathologic study of resected cases of adenosquamous carcinoma of the lung," Chest, vol. 109, no. 4, pp. 989-994, 1996.

[20] M. Wu, A. H. Szporn, D. Zhang et al., "Cytology applications of p63 and TTF-1 immunostaining in differential diagnosis of lung cancers," Diagnostic Cytopathology, vol. 33, no. 4, pp. 223-227, 2005.

[21] A. G. Nicholson, D. Gonzalez, P. Shah et al., "Refining the diagnosis and EGFR status of non-small cell lung carcinoma in biopsy and cytologic material, using a panel of mucin staining, TTF-1, cytokeratin 5/6, and P63, and EGFR mutation analysis," Journal of Thoracic Oncology, vol. 5, no. 4, pp. 436-441, 2010.

[22] G. Rossi, M. Papotti, M. Barbareschi, P. Graziano, and G. Pelosi, "Morphology and a limited number of immunohistochemical markers may efficiently subtype non-small-cell lung cancer," Journal of Clinical Oncology, vol. 27, no. 28, pp. e141-e142, 2009.

[23] A. Dejmek, P. Naucler, A. Smedjeback et al., "Napsin A (TA02) is a useful alternative to thyroid transcription factor-1 (TTF-1) for the identification of pulmonary adenocarcinoma cells in pleural effusions," Diagnostic Cytopathology, vol. 35, no. 8, pp. 493-497, 2007.

[24] L. M. Stoll, M. W. Johnson, E. Gabrielson, F. Askin, D. P. Clark, and Q. K. Li, "The utility of napsin-A in the identification 
of primary and metastatic lung adenocarcinoma among cytologically poorly differentiated carcinomas," Cancer Cytopathology, vol. 118, no. 6, pp. 441-449, 2010.

[25] J. Fukuoka, T. Dracheva, J. H. Shih et al., "Desmoglein 3 as a prognostic factor in lung cancer," Human Pathology, vol. 38, no. 2, pp. 276-283, 2007.

[26] V. Monica, P. Ceppi, L. Righi et al., "Desmocollin-3: a new marker of squamous differentiation in undifferentiated large-cell carcinoma of the lung," Modern Pathology, vol. 22, no. 5, pp. 709-717, 2009.

[27] D. Lebanony, H. Benjamin, S. Gilad et al., "Diagnostic assay based on hsa-miR-205 expression distinguishes squamous from nonsquamous non-small-cell lung carcinoma," Journal of Clinical Oncology, vol. 27, no. 12, pp. 2030-2037, 2009.

[28] J. A. Bishop, H. Benjamin, H. Cholakh, A. Chajut, D. P. Clark, and W. H. Westra, "Accurate classification of non-small cell lung carcinoma using a novel microRNA-based approach," Clinical Cancer Research, vol. 16, no. 2, pp. 610-619, 2010.

[29] V. D. Vescovo, C. Cantaloni, A. Cucino et al., "MiR205 expression levels in nonsmall cell lung cancerdo not always distinguish adenocarcinomas from squamous cell carcinomas," American Journal of Surgical Pathology, vol. 35, no. 2, pp. 268-275, 2011.

[30] H. Cheng, X. Xu, D. B. Costa, C. A. Powell, and B. Halmos, "Molecular testing in lung cancer: the time is now," Current Oncology Reports, vol. 12, no. 5, pp. 335-348, 2010.

[31] D. P. Clark, "Seize the opportunity: underutilization of fineneedle aspiration biopsy to inform targeted cancer therapy decisions," Cancer Cytopathology, vol. 117, no. 5, pp. 289-297, 2009.

[32] S. Sanati, D. W. Lu, E. Schmidt, A. Perry, L. P. Dehner, and J. D. Pfeifer, "Cytologic diagnosis of ewing sarcoma/peripheral neuroectodermal tumor with paired prospective molecular genetic analysis," Cancer, vol. 111, no. 3, pp. 192-199, 2007.

[33] N. K. B. Pang, S. Y. Chin, M. E. Nga et al., "Comparative validation of c-kit exon 11 mutation analysis on cytology samples and corresponding surgical resections of gastrointestinal stromal tumours," Cytopathology, vol. 20, no. 5, pp. 297-303, 2009.

[34] O. C. J. Schuurbiers, M. G. Looijen-Salamon, M. J. L. Ligtenberg, and H. F. M. Van Der Heijden, "A brief retrospective report on the feasibility of epidermal growth factor receptor and KRAS mutation analysis in transesophageal ultrasound- and endobronchial ultrasoundguided fine needle cytological aspirates," Journal of Thoracic Oncology, vol. 5, no. 10, pp. 1664-1667, 2010.

[35] J. H. Smouse, E. S. Cibas, P. A. Jänne, V. A. Joshi, K. H. Zou, and N. I. Lindeman, "EGFR mutations are detected comparably in cytologic and surgical pathology specimens of nonsmall cell lung cancer," Cancer Cytopathology, vol. 117, no. 1, pp. 67-72, 2009.

[36] M. E. Arcila, G. R. Oxnard, K. Nafa et al., "Rebiopsy of lung cancer patients with acquired resistance to EGFR inhibitors and enhanced detection of the T790M mutation using a locked nucleic acid-based assay," Clinical Cancer Research, vol. 17, no. 5, pp. 1169-1180, 2011.

[37] C. R. Kelsey, L. B. Marks, D. Hollis et al., "Local recurrence after surgery for early stage lung cancer: an 11-year experience with 975 patients," Cancer, vol. 115, no. 22, pp. 5218-5227, 2009.

[38] G. Sica, A. Yoshizawa, C. S. Sima et al., "A grading system of lung adenocarcinomas based on histologic pattern is predictive of disease recurrence in stage I tumors," The American Journal of Surgical Pathology, vol. 34, no. 8, pp. 1155-1162, 2010.
[39] D. E. Rudomina, O. Lin, and A. L. Moreira, "Cytologic diagnosis of pulmonary adenocarcinoma with micropapillary pattern: does it correlate with the histologic findings?" Diagnostic Cytopathology, vol. 37, no. 5, pp. 333-339, 2009.

[40] C. S. Sigel, D. E. Rudomina, N. Rekhtman, W. D. Travis, K. Geisinger, and A. L. Moreira, "Predicting Pulmonary Adenocarcinoma Outcome Based on a Cytology Grading System," Cancer Cytopathol, (In press).

[41] M. Brevet, M. Arcila, and M. Ladanyi, "Assessment of EGFR mutation status in lung adenocarcinoma by immunohistochemistry using antibodies specific to the two major forms of mutant EGFR," Journal of Molecular Diagnostics, vol. 12, no. 2, pp. 169-176, 2010.

[42] J. H. Paik, G. Choe, H. Kim et al., "Screening of anaplastic lymphoma kinase rearrangement by immunohistochemistry in non-small cell lung cancer: correlation with fluorescence in situ hybridization," Journal of Thoracic Oncology, vol. 6, no. 3, pp. 466-472, 2011.

[43] E. S. Yi, J. M. Boland, J. J. Maleszewski et al., "Correlation of IHC and FISH for ALK gene rearrangement in non-small cell lung carcinoma: IHC score algorithm for FISH," Journal of Thoracic Oncology, vol. 6, no. 3, pp. 459-465, 2011.

[44] J. Eckardt, K. E. Olsen, and P. B. Licht, "Endobronchial ultrasound-guided transbronchial needle aspiration of undiagnosed chest tumors," World Journal of Surgery, vol. 34, no. 8, pp. 1823-1827, 2010.

[45] M. A. Friedlander, C. S. Sigel, D. Finley et al., "Endobronchial ultrasound-guided transbronchial needle aspiration cytology - the memorial sloan-kettering cancer center experience," Cancer Cytopathology, vol. 118, no. 5, pp. 363-364, 2010.

[46] K. G. Tournoy, F. De Ryck, L. R. Vanwalleghem et al., "Endoscopic ultrasound reduces surgical mediastinal staging in lung cancer: a randomized trial," American Journal of Respiratory and Critical Care Medicine, vol. 177, no. 5, pp. 531-535, 2008.

[47] T. S. Weiser, K. Hyman, J. Yun, V. Litle, C. Chin, and S. J. Swanson, "Electromagnetic navigational bronchoscopy: a surgeon's perspective," Annals of Thoracic Surgery, vol. 85, no. 2, pp. S797-S801, 2008.

[48] B. Lamprecht, P. Porsch, C. Pirich, and M. Studnicka, "Electromagnetic navigation bronchoscopy in combination with PET-CT and rapid on-site cytopathologic examination for diagnosis of peripheral lung lesions," Lung, vol. 187, no. 1, pp. 55-59, 2009.

[49] G. Schreiber and D. C. McCrory, "Performance characteristics of different modalities for diagnosis of suspected lung cancer: summary of published evidence," Chest, vol. 123, no. 1, pp. 115S-128S, 2003.

[50] L. Santambrogio, M. Nosotti, N. Bellaviti, G. Pavoni, F. Radice, and V. Caputo, "CT-guided fine-needle aspiration cytology of solitary pulmonary nodules: a prospective, randomized study of immediate cytologic evaluation," Chest, vol. 112, no. 2, pp. 423-425, 1997. 


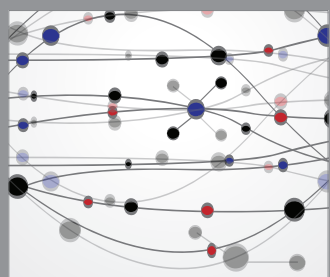

The Scientific World Journal
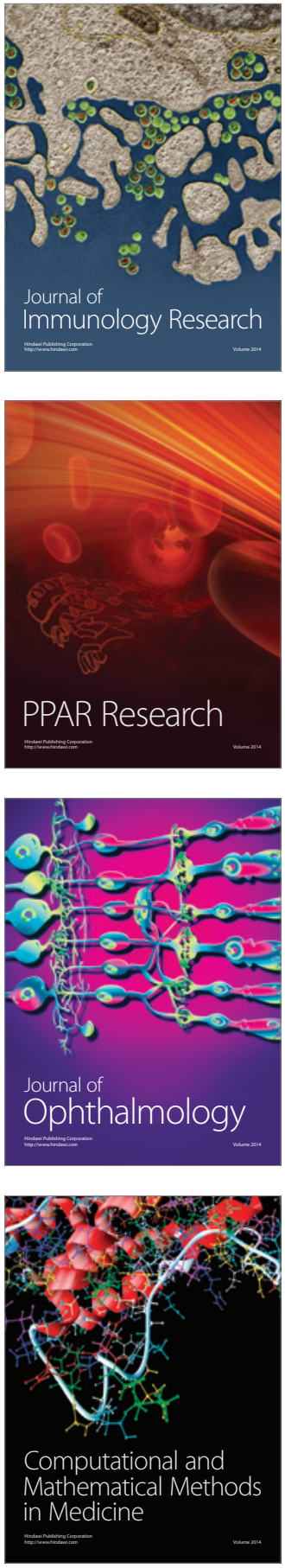

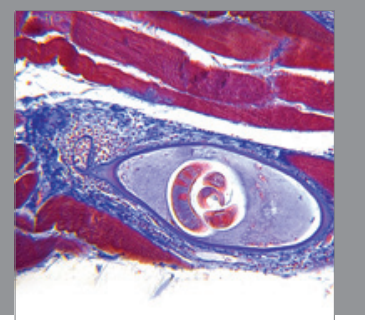

Gastroenterology

Research and Practice
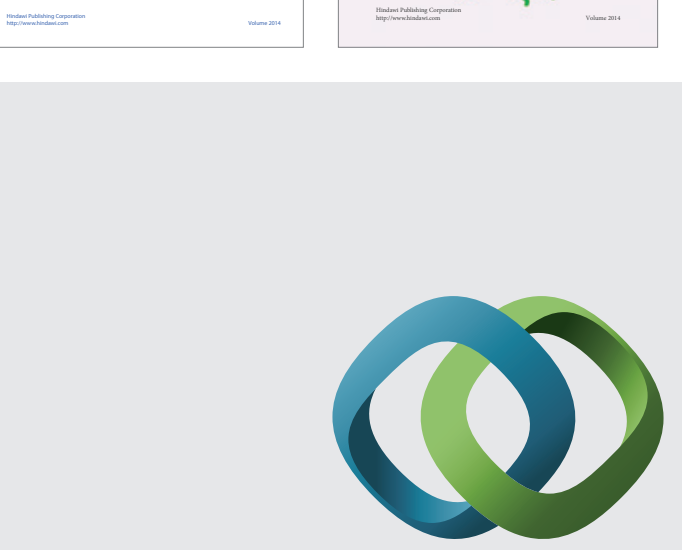

\section{Hindawi}

Submit your manuscripts at

http://www.hindawi.com
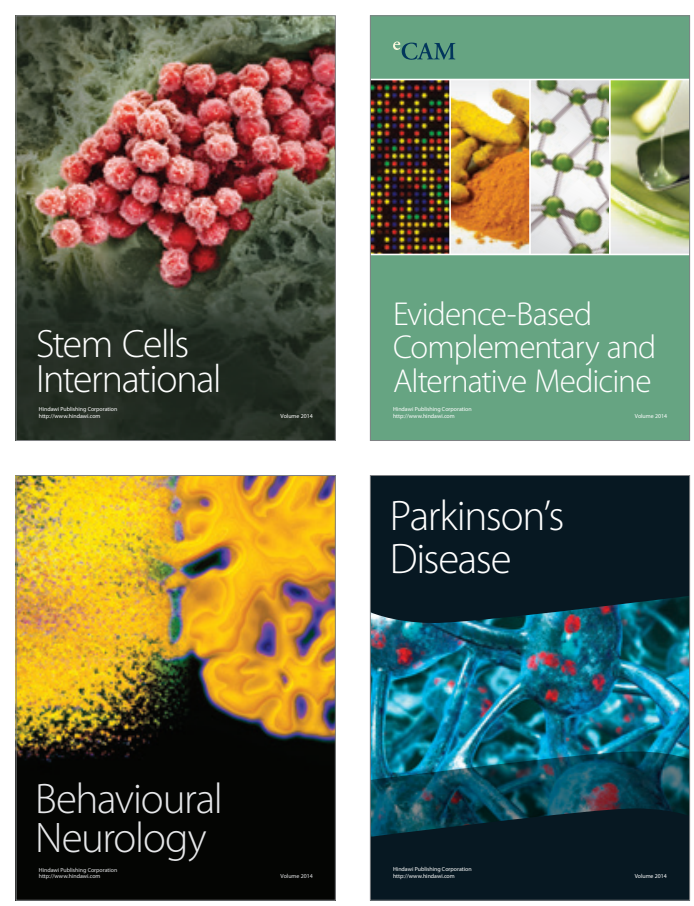

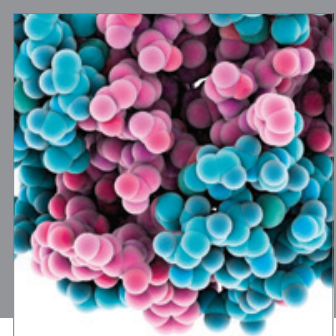

Journal of
Diabetes Research

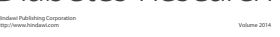

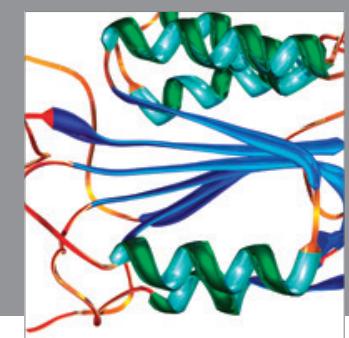

Disease Markers
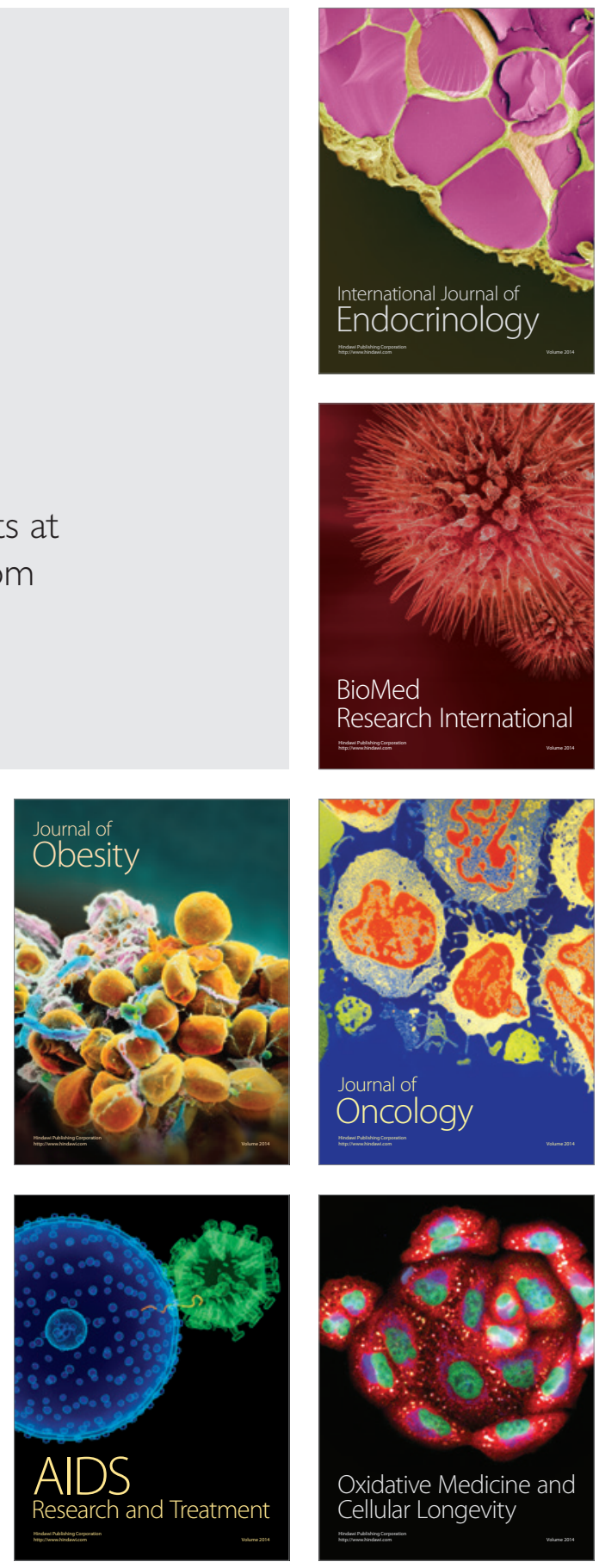